\title{
Influence of the emollient structure on the properties of cosmetic emulsion containing lamellar liquid crystals
}

Daria Terescenco $^{1}$, Celine Picard ${ }^{1}$, Florence Clemenceau ${ }^{2}$, Michel Grisel $^{1}$, Geraldine Savary ${ }^{1}$ *

${ }^{1}$ Normandie Univ, UNIHAVRE, FR 3038 CNRS, URCOM, 76600 Le Havre, France

${ }^{2}$ Direction Innovation - UID Excipients SEPPIC, 127 Chemin de la Poudrerie, 81105 Castres Cedex, France daria.terescenco@univ-lehavre.fr

celine.picard@univ-lehavre.fr

michel.grisel@univ-lehavre.fr

geraldine.savary@univ-lehavre.fr

florence.clemenceau@airliquide.com

* Corresponding author.

TEL: +33232743911 


\begin{abstract}
In the present study the effect of cosmetic emollients (oils and esters) varying from their chemical structure on the lamellar liquid crystals organization has been investigated. The cetearyl alcohol/cetearyl glucoside emulsifier, known for its ability to form lamellar phases was used at a level of $10 \%$ in emulsions. At this high concentration a strong lamellar network is formed. Also, each of the seven selected emollients was incorporated at level of $20 \%$, as for a classical cosmetic emulsion. These quantities were necessary in order to assure the presence of lamellar phases in all the samples and to amplify the impact of the emollient on the structural organization of the surfactant. Additionally, a control sample was formulated, containing only emulsifier and water. Emollients were divided as a function of their chemical structure in group A (without heteroatom) and B (with heteroatom). They were first described as a function of the interfacial tension against water, in order to classify them according to their hydrophobicity.

The presence of the lamellar phases in all samples was confirmed by means of optical microscopy under polarized light, and then the differences between emulsions physical properties were evidenced by static light scattering and rheological measurements. It was shown that use of A group emollients induces larger droplets size and, respectively, more elastic behavior to the emulsions. This phenomenon is related to the lamellar organization, which is dominated by $\alpha$-gel for A group oils and by lamellar liquid crystals combined to $\alpha$-gel for B group emollients, as showed through wide angle X-ray diffraction technique. Moreover, the influence of such structures on usage properties of emulsions was characterized via thermal analyses and spreadability tests.
\end{abstract}

\title{
Keywords
}

Emollient - interfacial tension - lamellar liquid crystals - $\alpha$-gel - Alkylpolyglucoside - surfactant -interaction

\section{Abbreviations}

APG: alkyl polyglucoside

APG/FA: alkyl polyglucoside/ fatty alcohol

CTFA: Cosmetic, Toiletry and Fragrance Association

DSC: differential scanning calorimetry

HLB: hydrophilic-lipophilic balance

HPLC: high-performance liquid chromatography

PMMA: poly(methyl methacrylate)

SLS: static light scattering

TDS: technical data sheet

WAXD: wide-angle X-ray diffraction 


\section{Introduction}

An emulsion is a system in which very fine droplets of a liquid are dispersed in a second liquid, the two liquids being partially or totally immiscible [1]. When considering cosmetic emulsions, which have esthetic standards to complete (texture, consistency, convenience to application, color, fragrance etc.), their composition becomes complex as consisting of several components like oil, water, surfactants, coloring agents, fragrances, preservatives, vitamins, etc. [2]. Each ingredient plays a role in the emulsion properties, making important the understanding of its individual properties, as well as the possible interactions with other ingredients.

Emollients are defined as "cosmetic ingredients which help to maintain the soft, smooth, and pliable appearance of the skin. Emollients function by their ability to remain on the skin surface or in the stratum corneum to act as lubricant, to reduce flaking, and to improve the appearance of the skin" by CTFA dictionary [3]. The first emollients for cosmetic use in the history were naturally occurring animal fats and vegetable oils.

Development of cosmetic industry required the improvement of the emollient's quality through increasing the stability (use of the antioxidants), reducing the characteristic odor and increasing their diversity by chemical modification and preparation of derivatives [4].

Lipophilic emollients are one of the most commonly used ingredients in cosmetic emulsions [5], represented by oils and fats from natural origins or obtained by chemical synthesis. As a function of their chemical structure, they are classified as "nonpolar" (paraffin's and isoparaffin's) and "polar" substances (esters and triglycerides), although this polarity parameter is seldom determined nor given [6].

Choosing the proper emollient for cosmetic formulation implies the necessity to understand its interaction with another main compound: the surfactant. Due to its amphiphilic structure, emulsifier molecule can form different structures in solutions, like liquid crystals, of hexagonal, lamellar or cubic type [7]. Liquid crystals possess liquid and solid properties at the same time as they are more organized than liquids, but less than solids [8], making them interesting for cosmetic use. Among other advantages, lamellar liquid crystals are similar to the stratum corneum lipidic structure [9] and they have a great skin hydration potential [10]. Also, liquid crystals can be involved in the emulsion stabilization process [11].

The APG (Alkyl PolyGlucoside) emulsifiers are able to form different structures, as it was showed by phases diagrams [12-14], from micelle towards lamellar phases. It is important to note that the formed structures are highly dependent on the chemical structure of APG (degree of polymerization, alkyl chain length, etc.). The situation changes, when a mixture of two surfactants is analyzed. In the cosmetic field, a mixture of two surfactants (of two different HLB values) is used in order to assure the better stability of emulsions. Meanwhile, adding a second molecule to the system surely changes its organization and a new phase diagram should be then established. Furthermore, a cosmetic emulsion should contain other ingredients in order to assure its sensorial properties. Consequently, the challenge of the research is to understand all the possible interactions between the surfactant molecules and other ingredients, and describe the effect of these compilations on the emulsifier's phase diagram.

The present research focuses on the investigation of possible interactions between the surfactant known for the lamellar liquid crystals formation (alkyl polyglucoside/fatty alcohol) $[10,15,16]$ combined with different kinds of emollients. First of all, seven emollients, well known in the cosmetic field, were selected and then described in terms of hydrophobicity, by measuring the interfacial tension versus water. Next, emulsions were formulated, containing $20 \%$ of emollient and $10 \%$ of emulsifier. The system was kept simple in order to highlight the interactions between the surfactant and the emollient. Two main objectives were then fixed. On the one hand, the effect of the emollient on the emulsions microstructure and macrostructure was described through droplets size and distribution measurements, rheological behavior determination, texture characterization, as well as thermal properties analyses. On the other hand, optical microscopy under polarized light, specific rheological tests and wide angle X-rays diffraction techniques were used to reveal the influence of the oil phase on the liquid crystals formation. 


\section{Materials and methods}

\subsection{Materials}

Cetearyl glucoside and cetearyl alcohol mixture, used as emulsifier, as well as the Phenoxyethanol and Methylparaben and Ethylparaben and Propylparaben and Butylparaben, used as preservative, were provided by SEPPIC, France. Seven emollients, liquid at room temperature and with similar viscosity, possessing different chemical structures, are presented in Table 1.

Table 1. INCI, trade name, the supplier, the codes and the viscosity values of seven chosen emollients

\begin{tabular}{ccccc}
\hline INCI & Trade name & Supplier & Codes & Viscosity (mPa.s) TDS \\
\hline Mineral oil & Huile Codex AAB2 & Aiglon & MO & 33 \\
Isohexadecane & Isohexadecane & IMCD & IHD & $6-8$ \\
Ethylhexyl palmitate & DUB PO & Stearinerie Dubois & EHP & $10-15$ \\
Ethylhexyl stearate & DUB SO & Stearinerie Dubois & EHS & $14-16$ \\
Caprylic/Capric Triglyceride & Myritol 318 & BASF & CCT & $27-33$ \\
Propylene glycol dicaprylate/ dicaprate & Myritol PC & BASF & PGDD & $9-12$ \\
Cocoglycerides & Myritol 331 & BASF & COCOG & $43-48$ \\
\hline
\end{tabular}

\subsection{Methods}

\subsubsection{Preparation of samples}

Seven emulsions were prepared as presented in Table 2. A control sample was also formulated. The control contained $10 \%$ of the same emulsifier, preservative and water, without oil addition. However it presented the structure of an emulsion, since the fatty alcohol from the emulsifier played the role of the dispersed phase.

Table 2. Composition of the formulated systems

\begin{tabular}{ccccc}
\hline & Surfactant & Oil phase & Aqueous phase & Preservative \\
\hline $\mathbf{7}$ oil containing emulsions & $10 \%$ & $20 \%$ & $69 \%$ & $1 \%$ \\
Control sample & $10 \%$ & - & $89 \%$ & $1 \%$ \\
\hline
\end{tabular}

Emulsions were prepared by heating $20 \%$ of the emollient and $10 \%$ of the emulsifier up to $75^{\circ} \mathrm{C}$ under mechanical stirring (400rpm), added to the water phase, also heated at $75^{\circ} \mathrm{C}$. For the control sample, $10 \%$ of emulsifier were heated up to $75^{\circ} \mathrm{C}$ and then added to the water phase at $75^{\circ} \mathrm{C}$. All the systems were then homogenized at $10,000 \mathrm{rpm}$ during one minute using a T25 digital ultra-turrax (IKA, Freiburg, Germany) equipped with the dispersing head S25N-25F. Next, mixtures were cooled down to $30^{\circ} \mathrm{C}$ under stirring (400rpm), and then the preservative was added. Once prepared, the products were divided into three samples, stocked at $4{ }^{\circ} \mathrm{C}, 40^{\circ} \mathrm{C}$ and ambient temperature, respectively, for stability monitoring.

\subsubsection{Physical characterization of emollients}

Density of each emollient was characterized with a portable densimeter Densito 30PX (Mettler Toledo, France).

Surface tension, as well as interfacial tension versus water, was measured with a Krüss K11 Tensiometer, using the Du Noüy ring method. A sufficient quantity of the emollient was introduced in the crystallizer to assure the height of the liquid equal to $11 \mathrm{~mm}$ minimum for the surface tension measurement. For the interfacial tension, $11 \mathrm{~mm}$ of water were covered with $11 \mathrm{~mm}$ (at least) of emollient to allow the interface formation. For the interfacial measurement versus water, both, push and pull methods were applied, adapted to each emollient. Measurements were carried out in duplicate.

The values of the surface tension and the interfacial tension versus water of each emollient were then collected. 


\subsubsection{Optical microscopy}

The emulsions microstructure was analyzed by means of a photomicroscope (Leica DMLP microscope) equipped with a camera: firstly, under the bright field and secondly, under the polarized light (using cross-polarizers) in order to investigate the presence of the liquid crystalline phase in each sample. Leica IM 1000 software was used to analyze the obtained micrographs.

\subsubsection{Droplet size distribution}

The emulsion droplets size were measured by static light scattering (SLS) using a laser diffraction particle size analyzer SALD-7500 nano (Shimadzu Co., Ltd, Japan) which is equipped with a violet semiconductor laser (405 nm) and a reverse Fourier optical system (the beam converge before encountering the sample). The emulsions were diluted in deionized water to achieve the absorption parameter equal to 0.2. Once introduced in the batch cell (7 $\mathrm{cm} 3$ ), the emulsions were stirred during the measurement to assure a homogenous dispersion. The dilution was necessary for destroying the droplet network and having access to the individual droplet size, without affecting the droplet itself. The nonappearance of coalescence induced by dilution was checked by optical microscopy. Data was analyzed using installed software, Wing SALD II and the droplets mean values for each emulsion were acquired.

\subsubsection{Rheological measurements}

Continuous and oscillatory measurements were performed using a controlled stress rheometer (HR1, TA instruments). A cone-plate aluminum device was used, with a diameter of $40 \mathrm{~mm}$, a cone angle of $1^{\circ} 59^{\prime} 38^{\prime \prime}$, and a gap of $47 \mu \mathrm{m}$. All the measurements were carried out in duplicate at $25^{\circ} \mathrm{C}$. For each test the sample was loaded with a spatula, and then the solvent trap was used to prevent sample drying. Once loaded, the samples were left at rest for two minutes prior to any measurement.

During continuous ramp testing, the flow properties were obtained by recording shear stress and viscosity at increasing shear rates ranging from 0.001 to $1000 \mathrm{~s}^{-1}$ (logarithmic mode) for $300 \mathrm{~s}$.

Linear viscoelastic region was obtained through oscillatory measurements conducted at $1 \mathrm{~Hz}$ with an increasing strain from $0.1 \%$ to $100 \%$ (logarithmic mode). The frequency sweep ramp was performed from 0.1 to $10 \mathrm{~Hz}$ at a constant strain, which was determined within the previously marked linear viscoelastic region of each sample. Then, storage (G') and loss moduli (G”), and tan $\delta$ (equal to ratio G'/G') were reported for the rheological characterization of investigated samples.

Measurements were carried out in duplicate.

\subsubsection{Wide-angle $X$-ray diffraction (WAXD)}

Samples were analyzed in reflection mode by X-ray diffraction (XRD) using a PANalytical Xpert Powder diffractometer (PANalytical B.V., Almelo, The Netherlands) with a $\operatorname{CoK}_{\mathrm{a}}(\lambda=1.7902 \AA)$ monochromatic radiation source, and operating voltage and current maintained at $40 \mathrm{kV}$ and $40 \mathrm{~mA}$, respectively. A $15 \mathrm{~mm}$ mask was used. The detector was a linear PIXcel1D detector. Data was acquired by steps of $0.02^{\circ}$ for $2 \theta$ values ranging from 2 to $50^{\circ}$. The formulations were pressed under Capton foil, avoiding air bubbles.

From diffraction angle theta $(\theta)$ the interlayer spacing were calculated according to Bragg's law.

\subsubsection{DSC - Differential scanning calorimetry}

Emulsions thermal properties were described by differential scanning calorimeter Pyris 1 DSC (PerkinElmer). Small amount of sample was weighted in $50 \mu \mathrm{L}$ aluminum pan and hermetically closed; meanwhile an empty aluminum pan was used as reference. As a function of product's type, the exact balanced quantities were not identical, ranging from 5 to $20 \mathrm{mg}$; for the pure emulsifier only $5 \mathrm{mg}$ were necessary, while for emulsions the quantity was increased up to $20 \mathrm{mg}$ to amplify the phase transitions thermal signature. The heating/cooling program was applied in the 
temperature range from $5^{\circ} \mathrm{C}$ to $90^{\circ} \mathrm{C}$ with the heating rate of $10^{\circ} \mathrm{C} / \mathrm{min}$. For the pure emulsifier, three consecutive heating/cooling cycles were applied, while emulsions sustained only one cycle.

Both the temperature corresponding to the peaks maxima $\left(\mathrm{T}^{\circ} \mathrm{C}\right)$ and the enthalpy values $(\Delta \mathrm{H} \mathrm{J} / \mathrm{g})$ of the each peak were collected.

\subsubsection{Texture analysis - spreading properties of emulsions}

Analysis of emulsions spreading properties was performed using a texture analyzer TAXT Plus (Stable Micro Systems, Cardiff, UK) in compression traction mode, equipped with friction module A/FR (ASTM-D 1894-90).

A polypropylene plastic sheet was fixed firmly to the base and the Helioplate TM HD 2 (Helioscreen, Marseille, France) plate, consisting of $50 \times 50 \mathrm{~mm}^{2}$ PMMA substrate and with homogeneous roughness on the entire surface, equal to $2 \mu \mathrm{m}$, is fixed to the sled. This sled is $63.5 \times 63.5 \mathrm{~mm}^{2}$ and weights $200+/-5 \mathrm{~g}$. $200 \mu \mathrm{L}$ of the cream was applied onto a $20 * 20 \mathrm{~mm}^{2}$ surface of the PMMA plate in four lines, parallel to the movement of the sled. The sled was then pulled across the polypropylene support at a constant speed $(3 \mathrm{~mm} / \mathrm{sec})$ on $60 \mathrm{~mm}$ and the force of the emulsion spreading was measured. PMMA plate and polypropylene sheet permitted to have adequate friction conditions while spreading the cream.

The area under the curve $(A+g . s e c)$, describing the difficulty to spread the product was then calculated. Three repetitions were performed for each sample.

\section{Results}

\subsection{Characterizing oils structure}

\subsection{1. $\log P$ calculation}

Emulsions oil phase is a complex system; it can be composed of various ingredients like hydrocarbons, triglycerides, esters, fatty alcohols etc.; all are different depending on their source, physical state or polarity. Hydrocarbons are considered as nonpolar oils, while polar oils are represented by fatty alcohols, esters and triglycerides, containing heteroatoms.

For this work seven cosmetic grade, lipophilic emollients were selected: nonpolar - mineral oil, isohexadecane; polar - cocoglycerides and caprilyc/capric triglycerides, as well as two similar esters - ethylhexyl palmitate and ethylhexyl stearate, of medium polarity. However the term "polarity" is still difficult to apply to all the ingredients. It can generally be determined for a single molecule, but not in case of a mixture (like mineral oil, cocoglycerides), for which the composition is not precisely known. That is why the literature does not give a real classification of cosmetic emollients as a function of their polarity.

We tried to classify the selected emollients by calculating $\log \mathrm{P}$. The octanol-water partition coefficient $\left(\mathrm{K}_{\mathrm{O}-\mathrm{w}}\right)$ is the ratio of a compound's concentration in octanol and in water at equilibrium at a specified temperature [17], $\log \mathrm{P}$ being the logarithm of the octanol/water partition coefficient. Often, this term is used in different domains like organic chemistry, pharmacy or analytical methods, like HPLC [18-20], in order to describe how hydrophilic or hydrophobic a chemical substance is. When $\log \mathrm{P}$ is small or inferior to zero, the molecule is considered hydrophilic. When $\log P$ increases, the hydrophobic character of the studied substance also increases.

The values of $\log \mathrm{P}$ of our five emollients (excluding mixtures: mineral oil and cocoglycerides), were calculated by four simulators [21], using the bond contribution method, or obtained from the chemical database [22] and presented in Table 3. One can observe that the obtained values are not homogeneous depending on the used simulator. Also, the results were not as expected. For example IHD, which is known to be nonpolar, possesses lower logP values than the esters. This phenomenon can be due to the octanol properties, it is not miscible with water, but in the same time it possesses a heteroatom. It means that IHD, consisting only of hydrocarbons is not compatible neither with water nor 
octanol, contrary to esters, which have more affinity with octanol. So, this technique is not suitable for emollient's classification.

Table 3. Calculated logP values of five pure emollients, calculated by four different simulators

\begin{tabular}{ccccc}
\hline Molecule & milogP & ACD/ $\log P$ & EPI suite $\log P$ & ChemAxon $\log P$ \\
\hline IHD & 6.43 & 7.98 & 7.79 & 6.51 \\
EHP & 9.27 & 11.15 & 10.61 & 9.55 \\
EHS & 9.52 & 12.21 & 11.59 & 10.31 \\
CCT & 6.7 & 6.9 & - & 3.59 \\
PGDD & 4.03 & 3.97 & - & - \\
\hline
\end{tabular}

Some patent's inventors [23,24], as well as some cosmetic ingredients suppliers evaluate the "polarity" by the values of interfacial tension between investigated oil and water. This technique is more suitable in case of emulsion formulation. Considering the emulsion properties, when oil phase is dispersed in water (continuous phase), the interface created between oil and water should be studied.

\subsubsection{Interfacial tension}

All the selected emollients have similar physical properties, liquid at room temperature, as well as quasi-transparent, which means that differences between them only occur at chemical level.

Emollients were classified in two groups:

Group A: MO and IHD composed only of hydrocarbon chains;

Group B: EHS, EHP, CCT, PGDD and COCOG, containing heteroatoms;

Table 4 summarizes the results of the density, surface tension and interfacial measurements. The density values were used to calculate surface and interfacial tensions. The results of surface tension for tested oils being very similar, the interfacial tension results were used to classify the emollients as a function of their hydrophobicity.

Table 4. Results of the density measurements, surface tension, as well as interfacial tension versus water of seven emollients

\begin{tabular}{cccc}
\hline Emollient & Density & Surface tension $(\mathbf{m N} / \mathbf{m})$ & Interfacial tension versus water $(\mathbf{m N} / \mathbf{m})$ \\
\hline MO & 0.847 & $30.02 \pm 0.02$ & $54.55 \pm 0.09$ \\
IHD & 0.787 & $24.40 \pm 0.03$ & $40.89 \pm 0.22$ \\
EHP & 0.859 & $29.65 \pm 0.10$ & $32.90 \pm 0.09$ \\
EHS & 0.859 & $29.83 \pm 0.13$ & $31.11 \pm 0.12$ \\
PGDD & 0.922 & $28.82 \pm 0.03$ & $22.99 \pm 0.16$ \\
CCT & 0.948 & $28.95 \pm 0.04$ & $13.72 \pm 0.04$ \\
COCOG & 0.936 & $29.54 \pm 0.01$ & $8.01 \pm 0.05$ \\
\hline
\end{tabular}

Following results will be respectively presented as a function of the established scale of the emollients hydrophobicity, based on the rank showed in Table 4.

The interfacial measurement was an adapted technique to compare such different raw materials from chemical and structural point of view. The obtained data are not perfectly transposable on the real model of the cosmetic emulsion, because it does not take into account the presence of the emulsifier. Meanwhile, it can give an idea of the emollient's behavior when interacting with the surfactant: we can suppose that MO and IHD will be more sensitive to surfactants alkyl chain; while CCT and PGDD could possibly sometimes interact with alcohol and glucose polar head. Consequently, different surfactant molecular organization is expected function of the used emollient.

Once the emollients characterized, the following step consisted in emulsion formulation. The composition was kept simple, containing only four ingredients: water, emollient, emulsifier and preservative, in order to discard the possible interactions between the ingredients and, consequently, to focus only on the emollient/emulsifier interaction. 


\subsection{Emollient impact on the emulsion properties}

\subsubsection{Effect of the emollient on the emulsions microstructure}

SLS analysis gave the information about oil in water emulsions droplet size, as well as their distribution: all the emulsions presented a monomodal polydisperse distribution, except the emulsion containing cocoglycerides, being less homogeneous, with plurimodal polydisperse distribution.

The differences between the emulsions are visible in terms of droplet median size. As presented in Figure 1, the use of A group oils provoked the formation of bigger oil droplets $(35-40 \mu \mathrm{m})$ in the system, than the use of B group ones $(5-10 \mu \mathrm{m})$. The maximum values for each emollient correspond to $90 \% \mathrm{D}(\mu \mathrm{m})$ of distribution and minimum to $10 \% \mathrm{D}(\mu \mathrm{m})$, showing that B group gave a monodisperse distribution of oil droplets, while the A group gave a polydisperse one.

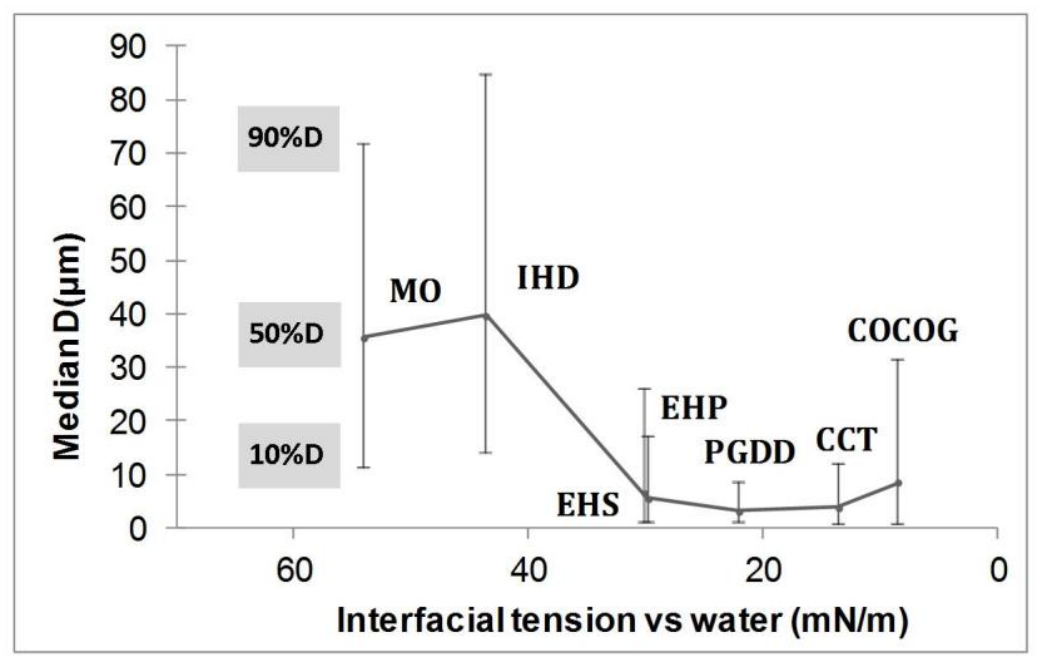

Figure 1. Median droplet size $(50 \% \mathrm{D})$ of emulsions as a function of the emollient interfacial tension versus water. Maximum and minimum values correspond to $10 \% \mathrm{D}$ and $90 \% \mathrm{D}$.

The results of particle size distribution analysis are complementary to microscopic observations. The micrographs of emulsions under the bright field (Figure 2) show the shape of obtained oil droplets, being distorted for A group oils and more spherical, as well as more homogeneous, for group B ones. 

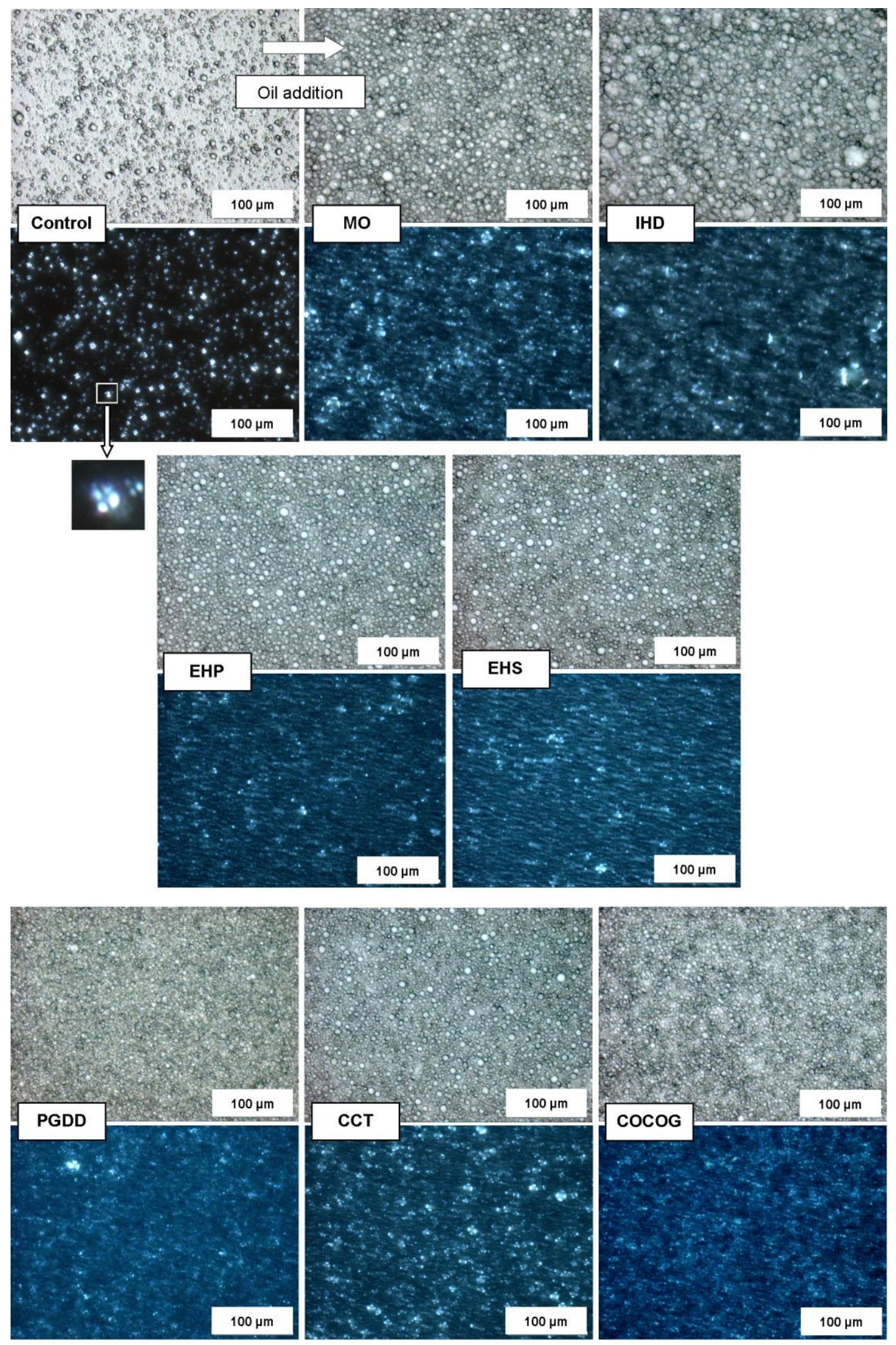

Figure 2. Bright field and polarized light micrographs of emulsions containing seven studied emollients. 
Microscopy confirmed SLS results, it can be even visually seen that there are differences between the droplet size as a function of the emollient group. Because the only modified parameter in all this examples is the emollients type, one can suppose that these microscopic differences are linked to the oils effect on the surfactant emulsification capacities. This first observation should confirmed by complementary techniques.

Another important information is obtained from the polarized light micrographs, showing that all the studied oil in water emulsions are birefringent (Figure 2). Specific textures, namely oily streaks combined with Maltese crosses, attest the presence of lamellar phases in the system [25,26]. These results indicate that lamellar liquid crystals obtained in control sample (only containing emulsifier and water), can be modified, but not totally destroyed (or annealed/hindered) by oil incorporation.

\subsubsection{Influence of the oil phase on the emulsions macroscopic structure}

\subsubsection{Rheology}

Rheological behavior of the studied emulsions was first analyzed by means of flow test. One can observe the differences of shear-thinning behaviors between A and B groups. Less viscous emulsions containing A group oils at low shear rates become more viscous than the B group ones at higher shear rates (Figure 3).

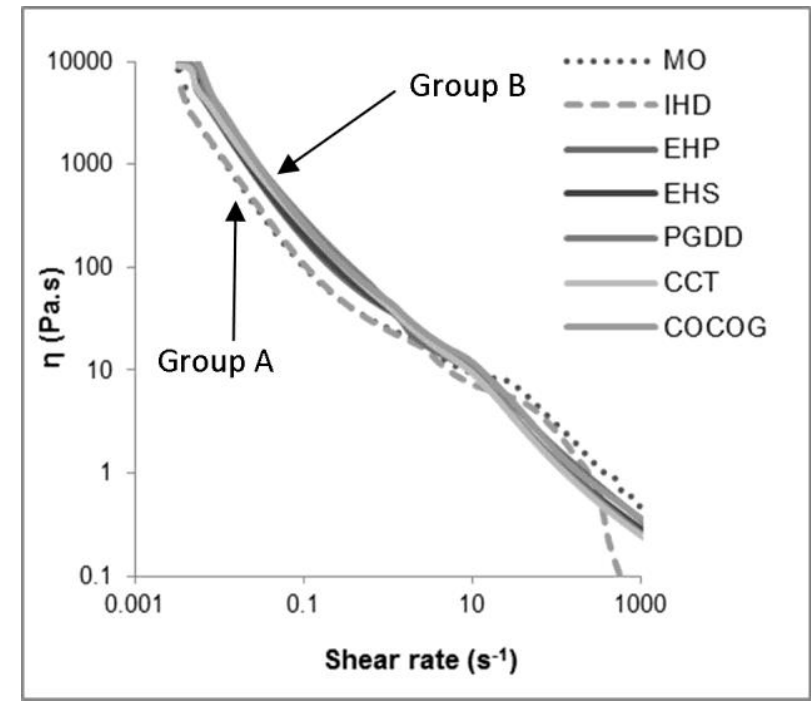

Figure 3. Viscosity as a function of shear rate for seven analyzed emulsions.

These results are in agreement with data obtained from the oscillatory test as for all the emulsions storage modulus G' remains higher than the loss modulus G", revealing the predominant viscoelastic behavior for all the products. Tan $\delta$ (equal to G"/G' ratio), which describes viscoelastic properties of the samples, possesses higher values for A group oils (Figure 4). Control sample was not included in these results, due to the impossibility to place it on the emollient's scale (based on the interfacial measurement versus water). 


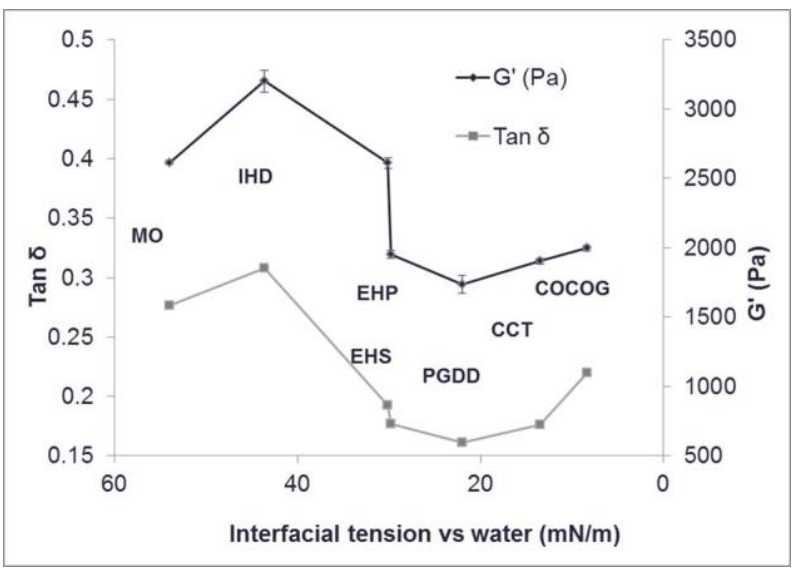

Figure 4. Storage modulus (G') and tan $\delta$ as a function of emollient interfacial tension versus water.

Rheological results are pointing clearly at the difference between the elastic and viscoelastic properties of two analyzed groups. The explanation to this phenomenon could be hidden in the specific organization of the emulsifier molecules influenced by the oil properties.

\subsubsection{Texture analysis}

Another technique used for describing the formulated emulsions was the spreading test. The same quantity of the product was spread under the same conditions and the area under the curve, expressing the difficulty to spread the sample, was recorded. Figure 5 presents data for seven emulsions containing emollients, as well as the control without emollient and the values of the spreading test of the sled on the PMMA support without any sample.

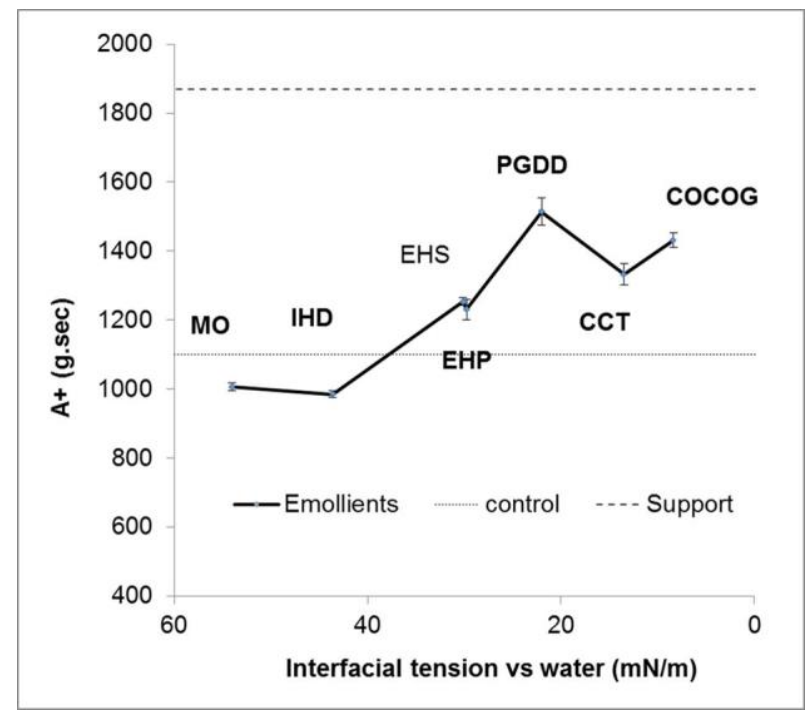

Figure 5. Difficulty of spreading (A+) of eight emulsions, including the control simple.

This is a relevant technique, complementary to other tests, because it is related to the perception of the cosmetic cream when applied on the skin. This test was realized in accordance to the protocol described in $[27,28]$ where it was proved that the spreading of emulsion, as evaluated by the evoked instrumental measurement, is significantly correlated with the sensory data obtained by an expert panel.

It considers the three steps of the product's evolution: spreading the emulsion, shearing it, and finally destroying it. The last step, when the emulsion structure is totally destroyed (droplets brake up), is governed by the direct interaction between the emulsions ingredients and the support (for the sensory analysis the support is represented by human skin). 
Figure 5 outlines the different behaviors of the emollients groups. On the one hand, emulsions from the group A are easily spread, possessing the values inferior to the control sample (without any oil phase). On the other hand, the group B requires bigger force than the control to be spread. Yet, the A+ (g.sec) for all the emulsions is always lower that the support test (without any sample).

One can suppose that the nonpolar support interacts with oil phase during the test, which means mineral oil and isohexadecane are easily spread, followed by the control sample without oil phase and then by oils from the group B. These results could be interpreted as a function of the known properties of the human skin. Accordingly to the studies [29,30], human skin is hydrophobic and even lipophilic if considering stratum corneum properties [31], which means that the results of the instrumental test could be very similar to the real application of the emulsions on the human skin.

\subsubsection{Emulsions thermal properties}

Previous experiments helped to establish that physical properties of the emulsions are influenced by the oil phase. The aim of DSC experiments is to bring further information about the emulsions thermal properties.

First the pure emulsifier was analyzed. In order to erase its thermal history three heating/cooling cycles were performed from $5^{\circ} \mathrm{C}$ to $90^{\circ} \mathrm{C}$. It was observed that the first heating cycle was different from the second and the third, the last two being identical. The results of the second cycle were taken into account considering that in case of emulsions, the emulsifier undergo the first heating cycle during emulsification, and the DSC analysis may correspond to its second heating cycle. This could be the explanation that in some studies of APG/FA emulsifier [32,33], the peak corresponding to alcohol $\beta-\alpha$ transition (which occurs at $36.6^{\circ} \mathrm{C}$ for cetostearyl alcohol [34]) is not observed for emulsions (see Table 5. Emulsifier's $1^{\text {st }}$ cycle versus $2^{\text {nd }}$ cycle).

The rest of the emulsions were analyzed by one cycle, because at $90^{\circ} \mathrm{C}$ their structure may be completely annealed. All data are gathered in Table 5. It shows all the observed peaks for each sample and the most significant peaks were shaded. The first observation is an important decrease of the enthalpy between the emulsifier and the control sample, containing the same emulsifier, since in the first case the emulsifier is pure and then it is "diluted" in the second case. Also, two clear peaks of cetearyl and cetearyl glucoside $\left(\mathrm{C}_{16}-\mathrm{C}_{18}\right)$ emulsifier are transformed in the single one for the diluted system (Figure 6). Consequently, one can affirm that that the surfactant is the control sample was not only diluted, but also completely reorganized, which results in specific thermal behavior.

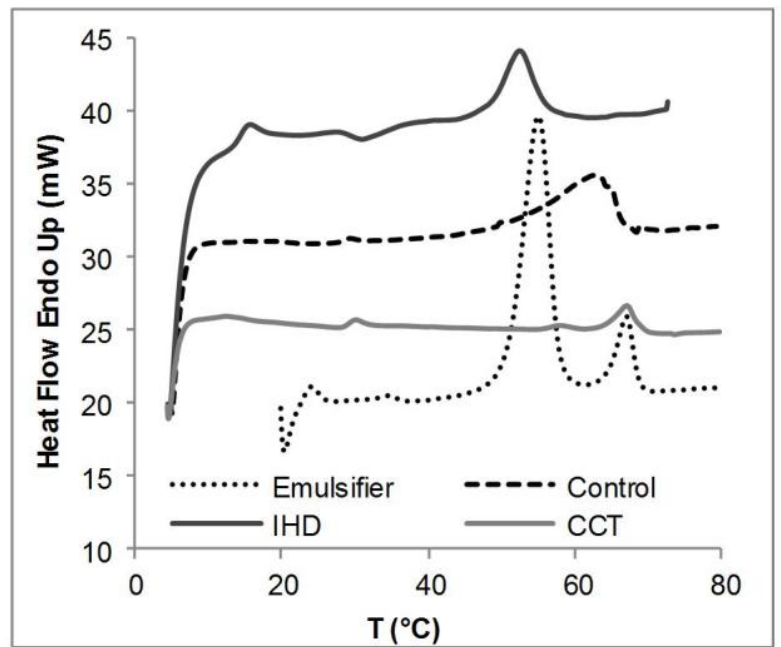

Figure 6. DSC profiles of pure emulsifier (2nd cycle), control sample, IHD (group A) and CCT (group B) emulsions. 
It is difficult to link the thermal response of the samples only to their structure. In reality several phenomena are involved, like the chemical structure of the molecules of all the ingredients, their interactions, the hydrocarbon chain length, etc. That's why the results are not directly related only to interfacial properties of the emollients.

The presented data suggest that the emollient addition can shift the main peak. A closer look to the two most important peaks (at $55^{\circ} \mathrm{C}$ and $65^{\circ} \mathrm{C}$ ) shows that the biggest enthalpies for MO, IHD, EHP intervene at $55^{\circ} \mathrm{C}$, while for PGDD, CCT, COCOG they are at $65^{\circ} \mathrm{C}$. The EHS result is disturbed by a peak at $9.52^{\circ} \mathrm{C}$, which should be the emollient solidification temperature.

Table 5. Results of DSC analysis, describing the $\mathrm{T}^{\circ} \mathrm{C}$ and $\Delta \mathrm{H} \mathrm{J} / \mathrm{g}$ of pure emulsifier, control sample and seven emulsions

\begin{tabular}{cccccccccc}
\hline \multirow{2}{*}{ Sample } & \multicolumn{2}{c}{ Peak 1 } & \multicolumn{2}{c}{ Peak 2 } & \multicolumn{2}{c}{ Peak 3 } & \multicolumn{2}{c}{ Peak 4 } \\
\cline { 2 - 9 } & $\mathbf{T}^{\circ} \mathbf{C}$ & $\Delta \mathbf{H} \mathbf{J} / \mathbf{g}$ & $\mathbf{T}^{\circ} \mathbf{C}$ & $\Delta \mathbf{H} \mathbf{J} / \mathbf{g}$ & $\mathbf{T}^{\circ} \mathbf{C}$ & $\Delta \mathbf{H} \mathbf{J} / \mathbf{g}$ & $\mathbf{T}^{\circ} \mathbf{C}$ & $\Delta \mathbf{H} \mathbf{J} / \mathbf{g}$ \\
\hline Emulsifier 1st cycle & & & 38.9 & 42.9 & 58.2 & 67.2 & 65.9 & 13.3 \\
Emulsifier 2nd cycle & & & 21.8 & 21.9 & 53.5 & 93.3 & 65.5 & 13.3 \\
Control & 15.3 & 0.5 & 29.4 & -0.1 & & & 62.7 & 12.7 \\
MO & & & 29.5 & 2.4 & 55.6 & 5.1 & 63.2 & 0.3 \\
IHD & 15.6 & 1.7 & 31.2 & -0.7 & 52.4 & 4.9 & & \\
EHP & 14.3 & 0.2 & 29.7 & -1.3 & 55.8 & 4.5 & 66.2 & 0.3 \\
EHS & 9.5 & 6.9 & 28.6 & -1.5 & 55.1 & 1.5 & 65.9 & 0.4 \\
PGDD & 14.1 & 0.8 & 30.7 & -1.2 & 54.6 & 0.3 & 65.3 & 2.5 \\
CCT & 12.6 & 0.7 & 30.2 & 0.2 & 57.9 & 0.3 & 67 & 2.6 \\
COCOG & & & 30 & -0.8 & 52.2 & 0.4 & 63.8 & 2.8 \\
\hline
\end{tabular}

Figure 6 shows how A group emulsions behave differently from control sample and B group emulsions through the temperature shift.

Previous results showed the effect of the emollient on the emulsions microstructure and macrostructure, as well as on its thermal properties. The next objective of this work consisted in understanding how the described oils affect the lamellar phase formation. For this purpose, complementary rheological tests and $\mathrm{X}$ rays analysis were carried out.

\subsection{Role of the oil phase in the lamellar liquid crystals formation}

\subsubsection{Rheology-frequency test}

The results of the frequency test are presented as G' and G' moduli as a function of frequency: for the emulsions containing A group Figure 7.a and B group oils Figure 7.b. For the seven emulsions storage modulus is parallel to loss modulus, corresponding to lamellar phase behavior [35-37]. Meanwhile, one can observe the differences in G" modulus behavior between two groups of oils which implies changes in $\tan \delta$ and, consequently, in viscoelastic properties of the samples as already shown. 


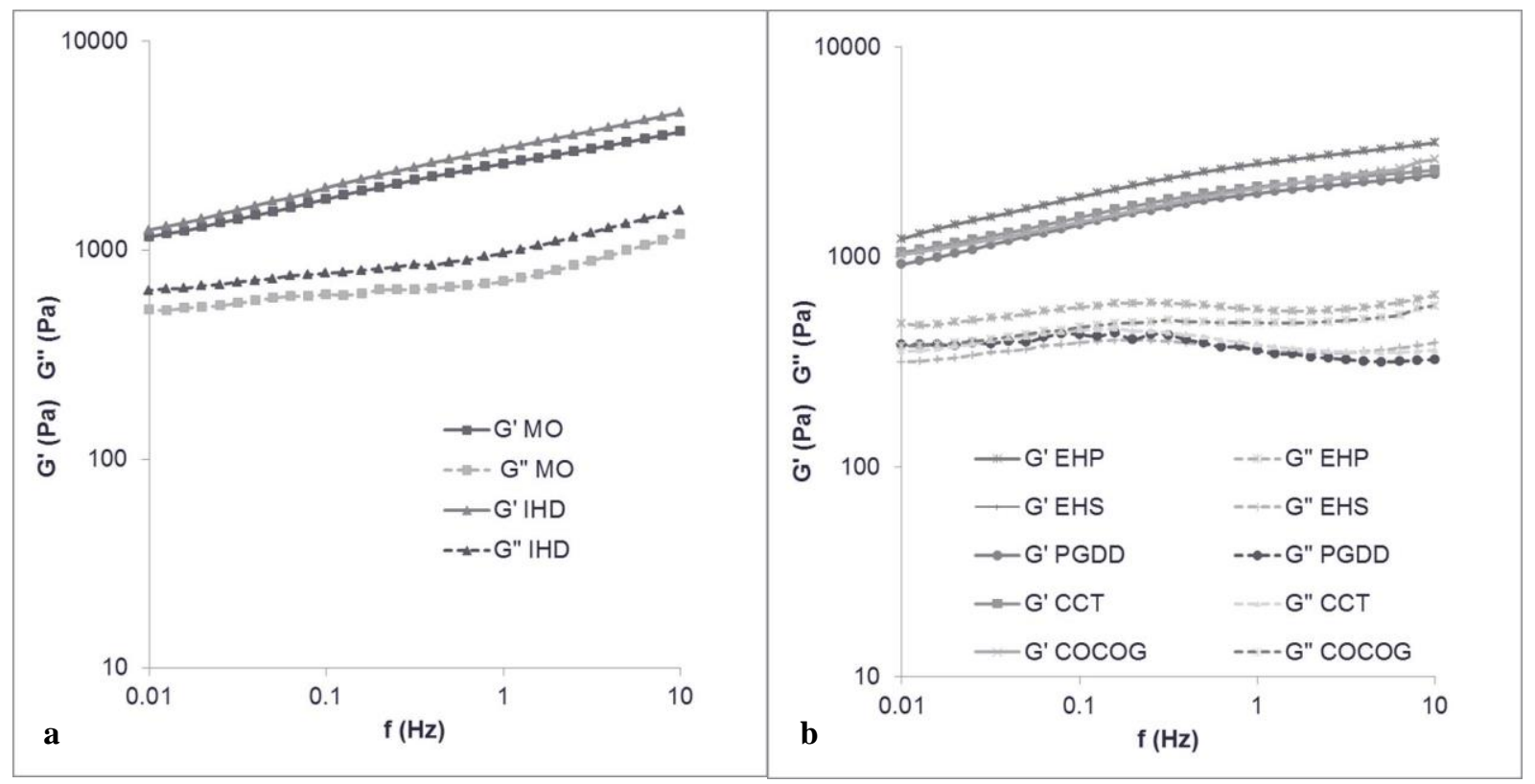

Figure 7. Frequency sweep test. Evolution of G' and G" moduli as a function of frequency. a) Emulsions containing A group oils; b) emulsions containing B group oils

For this reason the results of the frequency test were revised by adding the complementary data corresponding to the control sample and by observing the evolution of $\tan \delta$ as a function of frequency (Figure 8). The result was surprising and suggested that emulsion containing IHD and the control sample have the similar response to the solicitation, making them different from the B group one. Moreover, in this example the sample containing mineral oil behaves as intermediate between two groups: it has the same curve shape like IHD, but with smaller values for $\tan \delta$. These observations are complementary to the deformation test, because the control sample can clearly help to understand the link between the emulsion composition and its internal organization.

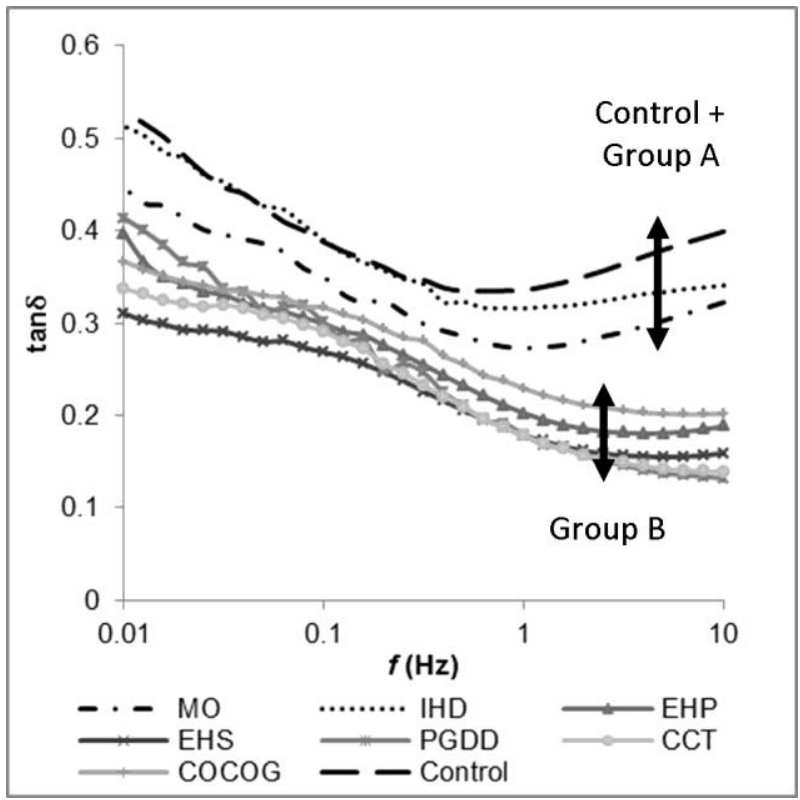

Figure 8. Frequency sweep test. Tan $\delta$ as a function of frequency for seven emulsions + control sample. 
To better understand these results, the WAXD (Wide-angle X-ray diffraction) technique was used for liquid crystals characterization. The previous conclusions require the control sample consideration in the understanding of the mechanism governing the liquid crystal formation

\subsubsection{WAXD - Wide-angle X-ray diffraction}

There exist different types of lamellar phases, $\mathrm{L}_{\alpha}, \mathrm{L}_{\beta}, \mathrm{L}_{\gamma}, \mathrm{L}_{\beta^{\prime}}, \mathrm{L}_{\delta}, \mathrm{L}_{\mathrm{s}}$, and $\mathrm{P}_{\beta^{\prime}}$, each being observed in lyotropic phase diagrams [38], but two main lamellar organizations are more current, namely lamellar liquid crystals $\left(\mathrm{L}_{\alpha}\right)$ and $\alpha$-gel $\left(\mathrm{L}_{\beta}\right)$. In the $\mathrm{L}_{\alpha}$ phase, the hydrocarbon chains are in a liquid-like state while in the $\mathrm{L}_{\beta}$ phase, the hydrocarbon chains remain stiff, perpendicular to the plane of the lamellae, with the axes organized as a two-dimensional hexagonal lattice [39]. Both organizations may be efficiently observed by WAXD (Figure 9 adapted from [40]).

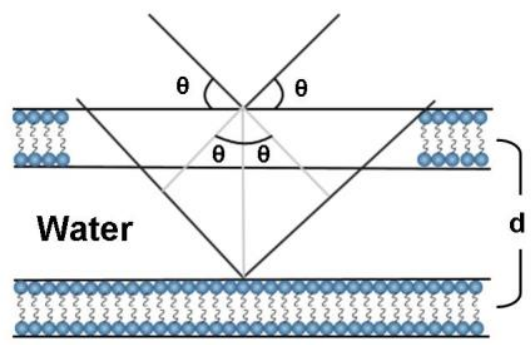

$2 \mathrm{~d} \sin \theta=\mathrm{n} \lambda$

Figure 9. Schematic diagram of X-ray diffraction of lamellar phase (adapted from [40])

For the $\alpha$-gel (with stiff hydrocarbon chains) the observed distance remains the same, with a clear sharp peak at the same value of $2 \theta$. In the meantime, for the lamellar liquid phase $\left(\mathrm{L}_{\alpha}\right)$, the hydrocarbon chains in the liquid-like state will have different lengths for different bilayers. Consequently, the response takes the shape of the diffuse halo, due to the differences in the observed distances and, respectively, in $2 \theta$ values.

Microscopic observations under the polarized light have already proved the presence of lamellar phases in all the samples, confirmed by the frequency test. However, the frequency test has also showed the differences between the emulsions containing A and B group emollients.

Figure 10 depicts the WAXD patterns of 4 samples: control, MO, IHD, CCT. All the emulsions from the B group (PGDD, CCT, COCOG, including the esters EHS, EHP) had similar pattern, that's why for simplicity only CCT emulsion was illustrated.

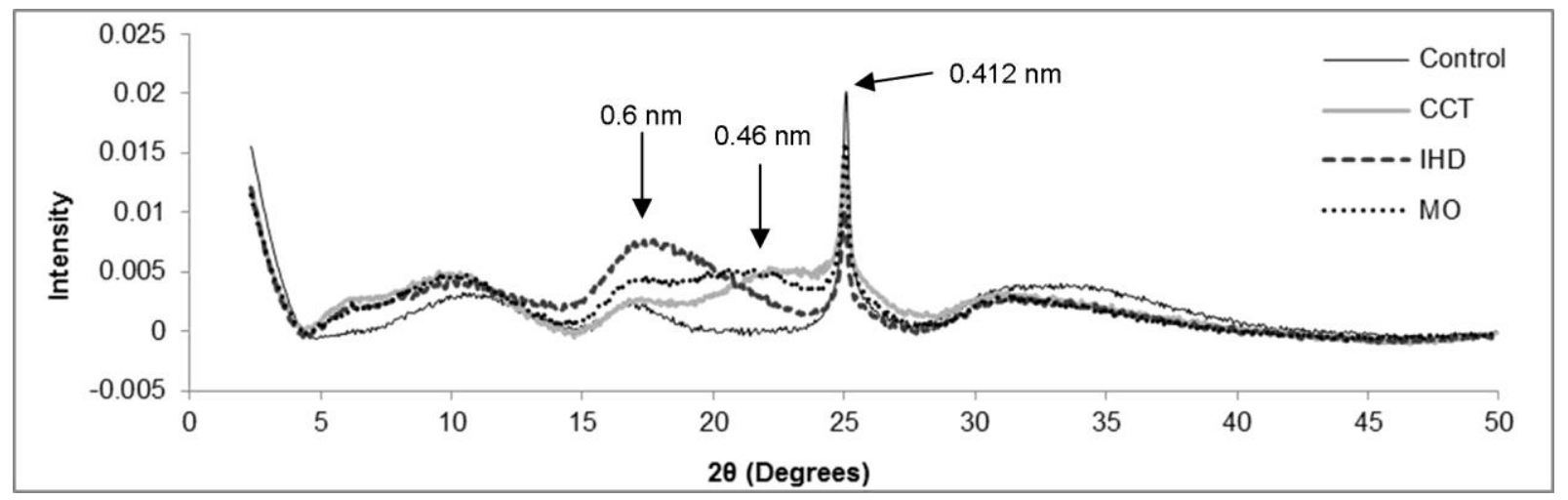

Figure 10. WAXD patterns of MO, IHD, CCT and control emulsions.

The main peaks can be described as diffuse halos thus illustrating a mainly amorphous like organization. This can be due to the matrix of the sample, containing mostly water as solvent. 
As it can be seen, the presence of $\alpha$-gel is clearly marked in all the samples by a corresponding sharp peak around $0.412 \mathrm{~nm}$ [26,41]; this peak outlines the rigid organization of $\alpha$-gel. In the same time, the presence of the lamellar liquid crystals $\left(\mathrm{L}_{\alpha}\right)$, with "fluid-like" characteristics, can also be evidenced at $0.46 \mathrm{~nm}[42,43]$.

This experiment demonstrates that $\alpha$-gel is formed in all the samples, with its center around $2 \theta=25^{\circ}$. Table 6 gives the properties of the $\alpha$-gel peak for all the emulsions, describing its height, its center and the calculated corresponding distance in $\mathrm{nm}$. This peak is the most intense for the control sample. Moreover, it is interesting to note that the diffuse halo at $0.46 \mathrm{~nm}$, which reflects the presence of lamellar liquid crystals in all B group samples, is less pronounced for MO, or even absent for the control sample and IHD. For these two, the diffuse halo is shifted towards $\mathrm{d}=0.6 \mathrm{~nm}$, describing significantly bigger repeated distance.

In the literature, Valoppi et al. [44] analyzed the organization of pure fatty alcohols with this technique; the sharp peaks observed at $0.42 \mathrm{~nm}$ and $0.41 \mathrm{~nm}$ were attributed to $\mathrm{C}_{18} \mathrm{OH}$ and the peak at $0.418 \mathrm{~nm}$ respectively to $\mathrm{C}_{16} \mathrm{OH}$. Moreover, the authors proposed the $\mathrm{X}$-rays diffraction patterns for the binary systems containing fatty alcohols and peanut oil as solvent. In case of low fatty alcohol concentration in the oil (5\%) a diffuse halo was observed between $15^{\circ}$ and $25^{\circ}$, similar with one peak observed in the present study $(0.6 \mathrm{~nm})$. As consequence, it can be suggested that the use of IHD as oil phase is more likely to interact with fatty alcohols by forming an organized structure, without achieving a lamellar liquid phase formation.

These results provide confirmatory evidence that chemical structure of the oil phase has a direct impact on the lamellar phase formation.

Table 6. Height, center and the calculated distance of each peak corresponding to $\alpha$-gel for eight analyzed samples

\begin{tabular}{cccc}
\hline Sample & Height & Center & d $(\mathbf{n m})$ \\
\hline Control & 0.0201 & 25.081 & 0.4123 \\
MO & 0.0158 & 25.028 & 0.4131 \\
IHD & 0.0101 & 25.028 & 0.4131 \\
EHP & 0.0152 & 25.024 & 0.4131 \\
EHS & 0.0143 & 24.923 & 0.4148 \\
PGDD & 0.0141 & 24.976 & 0.4139 \\
CCT & 0.0143 & 24.976 & 0.4140 \\
COCOG & 0.0116 & 25.186 & 0.4106 \\
\hline
\end{tabular}

These WAXD results can be related to the DSC analysis. Savic et al. [32] explain that for the polar emollients, the important decrease of enthalpies between the control sample (which contains $\alpha$-gel) and the emulsions is due to the insertion of medium alkyl chains within the mixed lipid bilayers causing the shift from ordered crystalline toward the liquid crystalline structures, formed at oil droplets border, while the crystalline gel dominates within the continual phase. This theory can be confirmed by our example through the apparition of the diffuse halo at $0.46 \mathrm{~nm}$ for B group emollients, corresponding to the lamellar liquid phase.

For the A group oils the situation is more complicated: the absence of the lamellar liquid phase in the IHD sample was proved by WAXD and confirmed by its unique behavior through rheology, DSC, SLS analysis, which means that its properties are influenced by $\alpha$-gel and maybe by complementary structures. When talking about the MO, its behavior is really similar to IHD, but slightly closer to the B group emollients. The explanation for this phenomenon may be hidden in the WAXD results: MO possesses both diffuse halos, at $0.6 \mathrm{~nm}$, like IHD, and at $0.46 \mathrm{~nm}$, meaning that it also contains a quantity of lamellar liquid crystals. This could be due to the fact that mineral oil is composed of hydrocarbons with alkyl chains of different lengths, disturbing the $\alpha$-gel organization. 


\section{Conclusion}

The present research was focused on the understanding of the emollients impact on the lamellar liquid crystal organization.

The control sample, without any oil phase, exclusively showed the presence of the $\alpha$-gel in the system. The $\alpha$-gel was disturbed by the oil phase addition. In the case of B group emollients (PGDD, CCT, and COCOG) and esters (EHS, EHP), lamellar liquid crystal formation around the droplets was observed, affecting the emulsions properties such as droplet size, shape and distribution, viscoelastic characteristics and spreading properties. In case of A group emollients, the formation of the lamellar liquid phase was less present (MO) or totally absent (IHD). The oil droplets of these samples have bigger size and they are less homogeneous, the elastic modulus is more pronounced, suggesting the idea of the $\alpha$-gel's strong influence on the system.

These results provide confirmatory evidence that heteroatom-free oils are more likely to keep the $\alpha$-gel organization in the system. However, it is important to consider the induced consequences: first, near to $\alpha$-gel, other structures were created inside the system (with repeated distance equal to $0.6 \mathrm{~nm}$ ). In addition, $\alpha$-gel, developed in the continuous phase, is less "protecting" the oil droplets, resulting in bigger elastic modulus, but in the same time in quicker system's destruction under strain. Finally, the thermal results showed quicker destabilization of the system occurring at $55^{\circ} \mathrm{C}$, contrary to heteroatom containing oils, destabilized at $65^{\circ} \mathrm{C}$, except the esters.

So, the results of the research did globally respect the emollient classification by interfacial tension versus water, but it is likely that the chemical structure of the emollient was crucial in the emulsions final properties. It can be suggested that the emollients containing heteroatoms actually form a synergy with the emulsifier, through the lamellar liquid phase formation around the droplets thus offering a more flexible, but more resistant structure inside the emulsion. 


\section{References}

[1] J. Knowlton, S. Pearce, Handbook of Cosmetic Science and Technology, Elsevier Advanced Technology, 1993.

[2] T.F. Tadros, Colloid Aspects of Cosmetic Formulations with Particular Reference to Polymeric Surfactants, in: T.F. Tadros (Ed.), Colloids Cosmet. Pers. Care, Wiley-VCH Verlag GmbH \& Co. KGaA, 2008: pp. 1-34.

[3] J.A. Wenninger, R.C. Canterbery, G.N. McEwen, T. and F.A. Cosmetic, International Cosmetic Ingredient Dictionary and Handbook, Cosmetic, Toiletry, and Fragrance Association, 2000.

[4] M. Berdick, The role of fats and oils in cosmetics, J. Am. Oil Chem. Soc. 49 (n.d.) 406-408. doi:10.1007/BF02582522.

[5] M.E. Parente, A. Gámbaro, G. Ares, Sensory Characterization of Emollients, J. Sens. Stud. 23 (2008) 149161. doi:10.1111/j.1745-459X.2007.00136.x.

[6] G. Zocchi, Skin Fel Agents, in: A.O. Barel, M. Paye, H.I. Maibach (Eds.), Handb. Cosmet. Sci. Technol. Second Ed., 3rd ed., CRC Press, 2005: pp. 357-370.

[7] T.F. Tadros, Phase Behaviour of Surfactant Systems, in: Appl. Surfactants, Wiley-VCH Verlag GmbH \& Co. KGaA, 2005: pp. 53-72

[8] P.J. Collings, M. Hird, Introduction to Liquid Crystals: Chemistry and Physics, CRC Press, 1997.

[9] Friberg S.E., Micelles, microemulsions, liquid crystals, and the structure of stratum corneum lipids, J Soc Cosmet Chem. 41 (1990) 155.

[10] S. Savić, M. Savić, S. Tamburić, G. Vuleta, S. Vesić, C.C. Müller-Goymann, An alkylpolyglucoside surfactant as a prospective pharmaceutical excipient for topical formulations: The influence of oil polarity on the colloidal structure and hydrocortisone in vitro/in vivo permeation, Eur. J. Pharm. Sci. 30 (2007) 441-450. doi:10.1016/j.ejps.2007.01.006.

[11] A.C.P. Duncke, T.O. Marinho, C.N. Barbato, G.B. Freitas, M.C.K. de Oliveira, M. Nele, Liquid Crystal Observations in Emulsion Fractions from Brazilian Crude Oils by Polarized Light Microscopy, Energy Fuels. 30 (2016) 3815-3820. doi:10.1021/acs.energyfuels.5b02943.

[12] D. Balzer, Alkyl polyglucosides, in: I.D. Robb (Ed.), Spec. Surfactants, Springer Netherlands, 1997: pp. 169207. http://link.springer.com/chapter/10.1007/978-94-009-1557-2_7 (accessed January 30, 2015).

[13] W. von Rybinski, Alkyl glycosides and polyglycosides, Curr. Opin. Colloid Interface Sci. 1 (1996) 587-597. doi:10.1016/S1359-0294(96)80096-3.

[14] M.W. Sulek, T. Wasilewski, Tribological properties of aqueous solutions of alkyl polyglucosides, Wear. 260 (2006) 193-204. doi:10.1016/j.wear.2005.02.047.

[15] S.D. Savić, M.M. Savić, S.A. Vesić, G.M. Vuleta, C.C. Müller-Goymann, Vehicles based on a sugar surfactant: Colloidal structure and its impact on in vitro/in vivo hydrocortisone permeation, Int. J. Pharm. 320 (2006) 86-95. doi:10.1016/j.ijpharm.2006.04.019.

[16] S. Savic, S. Tamburic, B. Jancic, J. Milic, G. Vuleta, The Impact of Urea on the Colloidal Structure of Alkylpolyglucoside-Based Emulsions: Physicochemical and In Vitro/In Vivo Characterization, in: T.F. Tadros (Ed.), Colloids Cosmet. Pers. Care, Wiley-VCH Verlag GmbH \& Co. KGaA, 2008: pp. 259-274.

[17] R. Bazemore, Sample preparation, in: K. Goodner, R. Rouseff (Eds.), Pract. Anal. Flavor Fragr. Mater., John Wiley \& Sons, 2011.

[18] Y.V. Kazakevich, R. LoBrutto, HPLC for Pharmaceutical Scientists, John Wiley \& Sons, 2007.

[19] R.B. Silverman, M.W. Holladay, The Organic Chemistry of Drug Design and Drug Action, Academic Press, 2014.

[20] T. Hanai, HPLC: A Practical Guide, Royal Society of Chemistry, 1999.

[21] Molinspiration Cheminformatics, (n.d.). http://www.molinspiration.com/ (accessed September 7, 2016). 
[22] LookChem,look for chemicals all over the world., (n.d.). http://www.lookchem.com/ (accessed September 7, 2016).

[23] J. Nielsen, R. Kropke, Cosmetic or dermatological formulations containing glycerin, 20040258654, 2004. http://www.freepatentsonline.com/y2004/0258654.html (accessed October 19, 2015).

[24] S. Behrens, U. Meiring, A. Clausen, A. Bleckmann, J. Nielsen, Emulsion comprising 1,2-alkanediols and polar oil components, US7875653 B2, 2011. http://www.google.com.ar/patents/US7875653 (accessed October 19, 2015).

[25] C.C. Müller-Goymann, Liquid Crystals in Drug Delivery, in: J. Swarbrick, J.C. Boylan (Eds.), Encycl. Pharm. Technol. Vol. 20 - Suppl. 3, CRC Press, 2000.

[26] C.E. Fairhurst, S. Fuller, J. Gray, M.C. Holmes, G.J.T. Tiddy, D. Demus, J. Goodby, G.W. Gray, H.-W. Spiess, V. Vill, Lyotropic Surfactant Liquid Crystals, in: Handb. Liq. Cryst. Set, Wiley-VCH Verlag GmbH, 1998: pp. 341-392. http://onlinelibrary.wiley.com/doi/10.1002/9783527619276.ch7d/summary (accessed July 27, 2016).

[27] G. Savary, M. Grisel, C. Picard, Impact of emollients on the spreading properties of cosmetic products: A combined sensory and instrumental characterization, Colloids Surf. B Biointerfaces. 102 (2013) 371-378. doi:10.1016/j.colsurfb.2012.07.028.

[28] L. Gilbert, G. Savary, M. Grisel, C. Picard, Predicting sensory texture properties of cosmetic emulsions by physical measurements, Chemom. Intell. Lab. Syst. 124 (2013) 21-31. doi:10.1016/j.chemolab.2013.03.002.

[29] A.E. Khyat, A. Mavon, M. Leduc, P. Agache, P. Humbert, Skin critical surface tension, Skin Res. Technol. 2 (1996) 91-96. doi:10.1111/j.1600-0846.1996.tb00066.x.

[30] A. Elkhyat, P. Agache, H. Zahouani, P. Humbert, A new method to measure in vivo human skin hydrophobia, Int. J. Cosmet. Sci. 23 (2001) 347-352. doi:10.1046/j.0412-5463.2001.00108.x.

[31] C.K. Lee, T. Uchida, K. Kitagawa, A. Yagi, N.-S. Kim, S. Goto, Skin permeability of various drugs with different lipophilicity, J. Pharm. Sci. 83 (1994) 562-565. doi:10.1002/jps.2600830424.

[32] S. Savic, G. Vuleta, R. Daniels, C.C. Müller-Goymann, Colloidal microstructure of binary systems and model creams stabilized with an alkylpolyglucoside non-ionic emulsifier, Colloid Polym. Sci. 283 (2004) 439-451. doi:10.1007/s00396-004-1174-4.

[33] M. Lukic, I. Pantelic, R. Daniels, C. Müller-Goymann, M. Savic, S. Savic, Moisturizing emulsion systems based on the novel long-chain alkyl polyglucoside emulsifier, J. Therm. Anal. Calorim. 111 (2012) 20452057. doi:10.1007/s10973-012-2263-0.

[34] L. Niemi, E. Laine, Effect of water content on the microstructure of an o/w cream, Int. J. Pharm. 68 (1991) 205-214. doi:10.1016/0378-5173(91)90143-C.

[35] M.A. Siddig, S. Radiman, L.S. Jan, S.V. Muniandy, Rheological behaviours of the hexagonal and lamellar phases of glucopone (APG) surfactant, Colloids Surf. Physicochem. Eng. Asp. 276 (2006) 15-21. doi:10.1016/j.colsurfa.2005.10.004.

[36] Z. Németh, L. Halász, J. Pálinkás, A. Bóta, T. Horányi, Rheological behaviour of a lamellar liquid crystalline surfactant-water system, Colloids Surf. Physicochem. Eng. Asp. 145 (1998) 107-119. doi:10.1016/S09277757(98)00576-7.

[37] M. Zheng, Z. Wang, F. Liu, Q. Mi, J. Wu, Study on the microstructure and rheological property of fish oil lyotropic liquid crystal, Colloids Surf. Physicochem. Eng. Asp. 385 (2011) 47-54. doi:10.1016/j.colsurfa.2011.05.040.

[38] A. Tardieu, V. Luzzati, F.C. Reman, Structure and polymorphism of the hydrocarbon chains of lipids: A study of lecithin-water phases, J. Mol. Biol. 75 (1973) 711-733. doi:10.1016/0022-2836(73)90303-3.

[39] A.M.F. Neto, S.R.A. Salinas, The Physics of Lyotropic Liquid Crystals: Phase Transitions and Structural Properties., Oxford University Press, 2005. 
[40] G.M. Eccleston, M.K. Behan-Martin, G.R. Jones, E. Towns-Andrews, Synchrotron X-ray investigations into the lamellar gel phase formed in pharmaceutical creams prepared with cetrimide and fatty alcohols, Int. J. Pharm. 203 (2000) 127-139.

[41] D. Kalnin, G. Garnaud, H. Amenitsch, M. Ollivon, Monitoring fat crystallization in aerated food emulsions by combined DSC and time-resolved synchrotron X-ray diffraction 论, Food Res. Int. 35 (2002) 927-934. doi:10.1016/S0963-9969(02)00155-2.

[42] R.P. Rand, V. Luzzati, X-Ray Diffraction Study in Water of Lipids Extracted from Human Erythrocytes, Biophys. J. 8 (1968) 125-137.

[43] K. Larsson, Crystal and liquid crystal structures of lipids, in: Food Emuls., Marcel Dekker Incorporated, 1976: pp. 39-66.

[44] F. Valoppi, S. Calligaris, A.G. Marangoni, Phase Transition and Polymorphic Behavior of Binary Systems Containing Fatty Alcohols and Peanut Oil, Cryst. Growth Des. 16 (2016) 4209-4215. doi:10.1021/acs.cgd.6b00145. 